\title{
LA TEORÍA DE LA EDUCACIÓN. OBJETO, ENFOQUES Y CONTENIDOS
}

\section{Educational Theory. Object, approaches and contents}

\section{La Théorie de l'Éducation. Objet, approches et contenus}

\author{
Bernardo GARGALLO LÓPEZ \\ Universidad de Valencia. Departamento de Teoría de la Educación. Facultad de \\ Filosofia y Ciencias de la Educación. Avda. Blasco Ibáñez, 30. 46010 Valencia. \\ Correo-e: bernardo.gargallo@uv.es
}

Fecha de aceptación definitiva: marzo de 2003

BIBLID [(1130-3743) 14, 2002, 19-46]

RESUMEN

En este artículo se intenta precisar el objeto y contenidos de la Teoría de la Educación entendida como disciplina científica. Se defiende su carácter de teoría para la praxis que debe integrar la dimensión contemplativa, "teórica", el conocimiento científico, y la dimensión práctica, de modo que ese conocimiento sirva como base para normativizar la praxis. Se interpreta la Teoría de la Educación como una teoría sustantiva del proceso educativo general que debe ocuparse también de otras cuestiones: conceptuales, epistemológicas, metodológicas, etc. La visión del autor con respecto al objeto de estudio se contrasta con diversas fuentes: trabajos de investigación previos, seminarios y congresos, revistas de Teoría de la Educación españolas, manuales de Teoría de la Educación y programas de la asignatura. El artículo concluye con una propuesta de temáticas fundamentales que se postulan como objeto de reflexión y de investigación científica para los teóricos de la educación.

Palabras clave: Teoría de la Educación, integración teoría-praxis, temas de investigación en Teoría de la Educación. 


\section{SUMMARY}

In this article we attempt to specify the object and contents of the Theory of Education interpreted as a scientific discipline. We defend its character of theory for practice which must integrate the contemplative climension, "theoretical", the scientific knowledge, and the practical dimension, so that that knowledge serves as a base for establishing norms for practice. We interpret the Theory of Eclucation as a substantive theory of the general educational process that also must study other problems: conceptual, epistemological, methodological, and so on. We contrast our vision with respect to the object of study with various sources: previous investigation works, seminars and congresses, spanish reviews of Theory of Education, textbooks of Theory of Education and curricular programmes of the subject. The article concludes with a proposal of fundamental themes that are postulated as the object of reflection and of scientific investigation for the theoreticians of the education.

Key words: Educational Theory, Theory of Education, integration of theory and practice, topics of research in Theory of Education.

\section{SOMMAIRE}

Dans cet article nous essayons de spécifier l'objet et les contenus de la Théorie de l'Éducation interprétée comme une discipline scientifique. Nous défendons son caractère de théorie pour la praxis qui doit intégrer la dimension contemplative, "théorique", la connaissance scientifique, et la dimension pratique, de sorte que cette connaissance serve comme base pour établir des normes pour la praxis. Nous interprétons la Théorie de l'Éducation comme une théorie substantielle du processus éducatif général qui doit étudier aussi d'autres problèmes: conceptuelles épistémologiques, méthodologiques, etc. Nous contrastons notre vision en ce qui concerne l'objet d'étude avec de diverses sources: travaux précédents de recherche, séminaires et congrès, revues de Théorie de l'Éducation espagnoles, manuels de Théorie de l'Éducation et programmes curriculaires de la matière. L'article conclut avec une proposition de thématiques fondamentales qui sont postulés comme objet de réflexion et de recherche scientifique pour les théoriciens de l'éducation.

Mots clef: Théorie de l'Éducation, intégration théorie-praxis, thématiques de recherche en Théorie de l'Éducation.

\section{InTroducción. La TeOría de la EdUCACIÓN COMO Disciplina CIENTífica}

Cuando hablamos de Teoría de la Educación en este artículo, la entendemos como disciplina científica o disciplina pedagógica sustantiva-teoría sustantiva en 
términos de Castillejo (1987) y de Touriñán (1987) ${ }^{1}$. Una disciplina, entendida en este sentido, cubre un sector del conocimiento científico - en nuestro caso se trata de una ciencia pedagógica en el universo de las Ciencias de la Educación-, se refiere a un objeto de conocimiento propio y bien delimitado, incluye un sistema articulado de conocimientos — conceptos, supuestos, principios, postulados, teorías, leyes...- y dispone de una metodología científica de indagación y aproximación al objeto ajustada a las características del mismo. No es objetivo de este artículo justificar el carácter de disciplina científica de la Teoría de la Educación, labor ya realizada por los investigadores del área desde que en los años 70 se decidiera adoptar el rótulo disciplinar de Teoría de la Educación entroncando con la tradición anglosajona, a partir de la conocida obra de Moore publicada en español en 1980. Para confirmarlo se pueden consultar diversas publicaciones fruto del trabajo de los Seminarios Interuniversitarios del área, que se celebran anualmente desde 1982 (http://www.ucm.es/info/site/), y también los artículos y manuales - a los que luego se hará mención- editados sobre el tema.

En todo caso, conviene subrayar que esta disciplina pertenece al ámbito de las ciencias sociales y los problemas de estatus científico que se pueden plantear con respecto a ella son los mismos que afectan a las otras ciencias pedagógicas y a las otras ciencias sociales, que no responden al modelo de ciencia "dura", el de las ciencias físico-naturales, precisamente por la complejidad del objeto del que se ocupan - en el caso de las ciencias pedagógicas, la educación-, y que han desarrollado métodos adaptados al objeto de estudio. Como afirma Bunge, «el método científico no se rompió cuando se estiró para que abarcara los problemas sociales. Tampoco se rompe si se lo aplica a otras disciplinas, en particular las humanísticas" (Bunge, 1980, 42). Las ciencias físicas son sólo una especie dentro del género más amplio de la ciencia. Cada ciencia tiene su propio sistema de enunciados, su propio lenguaje y sus modos de acceso a la realidad en que incide (Medina, 2000b). De hecho, en Teoría de la Educación coexisten metodologías diversas, unas de corte hermenéutico-interpretativo y socio-crítico (cualitativas) y otras de corte empíricoanalítico (cuantitativas) —en menor grado- con métodos reflexivo-discursivos y analógicos (García Carrasco y García del Dujo, 1995; López-Barajas, 2000; Martínez y Buxarrais, 1992; Medina, 2000c).

1. La Teoría de la Educación, al igual que otras ciencias "pedagógicas", se distingue de las otras Ciencias de la Educación (ciencias humanas y también otras ciencias. que se han aplicado al problema educativo: Psicología de la Educación, Sociología de la Educación, Economía de la Educación, Biología de la Educación, etc.), que no son teorias "Sustantivas" de la educación, sino teoriáa "interpretativas" (CASTILlEIO, 1987; TOURIŃÁN, 1987). Otras denominaciones en la literatura para estas últimas son: teorías "acerca" de la educación (Moore, 1980), ciencias "auxiliares" (QuinTANA, 1983). teorías del "hecho educativo" (Trilla, 1987), o ciencias "fundamentantes" (Colon y RonRíGUEz, 1996). Las teorías "Sustantivas" son ciencias "pedagógicas", disciplinas que se generan única y exclusivamente por y para el objeto educación - no existirían sin él-: Pedagogía Social, Didácticil, Educación Especial. Teoría de la Educación, Educación Ambiental... 
En este artículo, nuestra reflexión se va a centrar en torno al objeto de estudio de la disciplina y a los contenidos que en ella se abordan para sacar conclusiones que puedan estimular el debate intelectual.

Desde nuestro punto de vista, la Teoría de la Educación entendida como disciplina científica, como teoría sustantiva de la educación, tiene como objeto describir, explicar, interpretar, comprender (dimensión "teórico-científica") y transformar (dimensión "normativo-tecnológica") el proceso educativo general. Es ésta una interpretación compartida por diversos teóricos (Medina, 2000a; Sarramona, 2000; Touriñán, 1987). En ese sentido, podría interpretarse como una teoría sustantiva global del proceso educativo, aunque no sea "la" teoría global de la educación ${ }^{2}$.

La Teoría de la Educación se nos presenta, pues, como un conjunto sistemático de conocimientos que se proyectan en la intervención pedagógica, de modo que saber científico y saber tecnológico ${ }^{3}$ son formas de saber que se integran en la Teoría de la Educación de forma inseparable (Castillejo, 1987; Castillejo y Colom, 1987; Colom, 1986, 1997; García Carrasco, 1983 y 1987; García Carrasco y García del Dujo, 1996; Medina, 2000b; Puig, 1986 y 1989; Sarramona, 1990, 1992 y 1995; Tourinaán, 1987; Vázquez, 1994). Nuestra concepción de la Teoría de la Educación, disciplina científica, es, pues, científico-tecnológica, como lo es de las demás disciplinas pedagógicas, que desde nuestro punto de vista están orientadas a la acción, a la normativización de la acción educativa. En eso se distinguen de las otras Ciencias de la Educación (teorías "interpretativas"), que aportan conocimiento científico pero cuyos intereses fundamentales no son normativos, sino descriptivoexplicativos.

Por otra parte, éste es el sentido de la Teoría de la Educación que descubrimos si seguimos la pista de su origen (Colom, 1997). En los años 70, se fue abriendo paso en el profesorado de Pedagogía General la corriente anglosajona que consideraba a la Teoría de la educación como la fundamentación científica de la práctica educativa, mientras que la tradición germánica de la "Pedagogía” vigente en nuestro país era de índole más especulativa y filosófica, y no estaba tan preocupada por la práctica (Sarramona, 2000). En el mundo anglosajón la Teoría de la Educación es teoría para la práctica de la educación. Sería absurdo, en ese sentido, concebir una teoría educativa que no pueda incidir en la práctica. En este

2. Las progresivas desmembraciones de la Pedagogía General herbartiana (CoLOM, 1997) nos han conducido al universo de las Ciencias de la Educación que abordan el objeto — complejo- desde diversas perspectivas, y la Teoría de la Educación lo hace desde la suya.

3. Entendemos la tecnología no como la pura técnica o ciencia aplicada (BUnGe, 1981; CASTILLEJO y Colom, 1987), sino como "teoría de la técnica", ya que la tecnología - que integra la techné: saber hacer con conocimiento de causa, distinto de la empeiria o saber hacer por mera habilidad a partir de la propia experiencia, y el logos: reflexión sobre la técnica, teoría o tratado de la técnica (Fullat, 1991) implica someter a reflexión teórica el problema de acción, que se busca resolver mediante la aplicación de técnicas, y también las leyes científicas que las sustentan (GARCía ARETIO, 2000), siendo sus características más definitorias la racionalidad, el sistematismo, la planificación, la claridad de metas, el control, la eficacia y la optimización (SARRAMONA, 1990). 
contexto, la teoría se entiende como un "cuerpo sistemático y coherente de conocimientos con capacidad de propiciar en su aplicación efectos esperados; o sea, que más o menos, si bien sin el rigurosismo epistemológico de la filosofía de la ciencia, "teoría" se puede adscribir a "ciencia", al menos en el sentido con que se reconoce a las teorías en las actuales ciencias humanas" (Colom, 1997, 148).

La Teoría de la Educación que postulamos integrará, pues, el conocimiento ${ }^{4}$ de la realidad educativa de cara a la intervención sobre la misma para mejorar la práctica (Colom y Núñez Cubero, 2001). No se limitará, sin embargo, a ser una ciencia de síntesis o "de acarreo" sin más, que se limite a recoger tales aportaciones, dado que, con ellas, ha de producir un pensamiento teórico y nuevo que las dote de unidad y aborde el hecho educativo en su integridad y complejidad. La "Teoría de la Educación, pues, sólo tiene sentido si es teoría para la práctica; o, si se quiere, saber para bacen (Colom, 1997, 149).

Colom (1997) realiza, desde nuestro punto de vista, una excelente síntesis de lo que sería la estructura conceptual de la Teoría de la Educación, que vendría dada por tres niveles de conocimiento que ayudarian a acotar el objeto:

1. Nivel previo de carácter metateórico: que integraría la concepción de la realidad (qué sea la educación), el análisis del modo de conocimiento de esa realidad (epistemología), y también de las formas de acceso a esa realidad (procesos heurísticos y metodologías de investigación).

2. Nivel teórico-científico: que integraría las propias aportaciones, las de las Ciencias de la Educación y de otras disciplinas que se ocupen del objeto de estudio a fin de conocer científicamente (describir, interpretar, explicar, comprender) la realidad educativa sobre la que se ha de intervenir.

3. Nivel tecnológico-aplicativo: a partir del conocimiento disponible se trata de normativizar la acción educativa y de convertirla en pedagógica (procesos de intervención, mejora, optimización, innovación).

En definitiva, la Teoría de la Educación constituye un conjunto sistemático de conocimientos que procurará la mejora de la práctica educativa mediante la proposición de normas de acción, lo que supondrá siempre ciertos niveles de concepción tecnológica.

\section{EL OBJETO DE ESTUDIO}

La Teoría de la Educación, heredera de la Pedagogía General, a la que de facto ha venido a sustituir en nuestro país, y de la tradición fundamental y sistemática,

4. En ese sentido, se trata de una disciplina eminentemente "oportunistan, que utilizará las aportaciones de conocimiento científico de las diversas disciplinas que le convengan y también las propias aportaciones, así como las metodologías que se ajusten a sus objetivos. 
pero con una orientación más empírica, y entendida como fundamentación científica de la práctica educativa (Marín, 1997), debe ocuparse de la educación en general, del proceso educativo general, de lo que sería, en palabras de Quintana $(1988,27)$, el "tronco común del árbol pedagógico", o el "núcleo típicamente pedagógico" en palabras de Marín $(1997,549)$. En el contexto de la ciencia pedagógica española, desde nuestro punto de vista, es ésta la única disciplina que puede ofertar un saber comprensivo y una visión de conjunto de la problemática educativa, necesaria para articular un marco coherente, integrador y fundamentante frente a la fragmentación de las diversas pedagogías y ciencias de la educación.

Por eso, la Teoría de la Educación se ha de ocupar de la intervención pedagógica general. Ése sería su objeto fundamental de indagación. Así, se podría entender como teoria sustantiva global del proceso educativo general, cuya tarea es la descripción, interpretación, explicación y comprensión del fenómeno educativo, del hecho y del proceso educativo ${ }^{5}$. Ello supone integrar los conocimientos necesarios para fundamentar la acción educativa — que se convertirá en pedagógica-. La construcción de una estructura interpretativa del proceso educativo permitirá la génesis de "teorías sustantivas parciales", que atienden a dimensiones específicas: Didácticas, de Orientación, de Pedagogía Social, etc., y las correspondientes derivaciones de tecnologías educativas específicas.

El carácter de disciplina científica integradora y fundamentante antes aludido hace necesario que la Teoría de la Educación aborde cuestiones epistemológicas referidas al modo de conocimiento científico-pedagógico ${ }^{6}$, a la propia Teoría de la Educación, a la delimitación del campo de las otras disciplinas pedagógicas y de las diversas ciencias de la educación y de la propia disciplina.

Del mismo modo, no se puede eludir, desde nuestro punto de vista, la referencia a las formas de acceder a ese conocimiento, lo que nos lleva a la reflexión

5. Al articular el objeto fundamental de estudio de la Teoría de la Educación en torno al "proceso educativo general. se evita, al menos en parte, el solapamiento que podría producirse con la Didáctica General que se ocupa de elementos de la teoría del currículo y de los componentes didácticos del proceso de enseñanza-aprendizaje -objetivos, programación, evaluación, etc.--, ubicados fundamentalmente en el ámbito del diseño instruccional referido al entorno escolar — sistema educativo regladoBasta con analizar los contenidos de obras cle Didáctica General (Rodríguez Diéglez, 1985; Sáenz, 1989) o de Teoría del Currículum (Angllo y Blanco, 1994; Gimeno y Pérez Gómez, 1992; Zabalza, 1997) para constatarlo. La propia Dicláctica General se solapa con la Psicología de la Instrucción en muchos de sus elementos. Y es que en Pedagogía falta una delimitación precisa de fronteras (Larrosa, 1990), tarea nada fácil, por otra parte, porque el trazado de esas fronteras no es sólo cuestión científica, sino "política" y de intereses. En todo caso nosotros nos ocupamos del "proceso educativo general", de educación -en ámbitos de educación formal, no formal e informal-y los didactas de enseñanza e instrucción, preferentemente en situaciones de educación formal.

6. Lo más habitual es que las "Teorías de la Educación” publicadas en nuestro país incluyan algún capítulo de tipo epistemológico aunque, en ocasiones, la temática concita tal interés que se convierte en culasi-exclusivo objeto de reflexión del teórico de la educación, como ocurre en las obras de LARRosa (1990) y COLOM (2002). 
sobre los procesos de investigación y sobre la metodología de investigación, tanto de la propia Teoría de la Educación como de la Pedagogía en general.

Por otra parte, para describir, comprender, explicar y normativizar el proceso educativo, la Teoría de la Educación, como disciplina pedagógica sustantiva o disciplina científica, debe atender a una serie de temas fundamentales que tiene que hacer también objeto de la propia reflexión teórica. Veamos aquellos que juzgamos más nucleares:

- La Teoría de la Educación, en cuanto que se ocupa del hecho educativo, debe integrar un cuerpo de conocimientos a propósito del objeto en que se pretende incidir (Puig, 1989). Un planteamiento tecnológico, no tecnicista, demanda necesariamente poseer con anterioridad una cierta cantidad de conocimiento sobre el qué de lo que se quiere mejorar y sobre su funcionamiento. Qué sea la educación y los procesos educativos son preguntas que la Teoría de la Educación se ha de contestar. La reflexión sobre el fenómeno y el hecho educativo es fundamental.

- Una Teoría de la Educación científico-tecnológica adquiere sentido vinculada a la dimensión teleológica y axiológica de la educación y la reflexión sobre la misma le es irrenunciable. La Teoría de la Educación, como teoría científico-tecnológica de la acción educativa, pretende fundamentalmente la optimización de dicha acción, convertida así en pedagógica, de cara a conseguir los fines o patrones propuestos. Si no se quiere caer en un puro utilitarismo, que reduciría nuestra disciplina a una teoría puramente instrumental (Barrio y Ruiz, 1992), al estilo tecnocrático o tecnicista (Gil Cantero, 1991), la reflexión sobre los fines es fundamental. La acción educativa se justifica por el conocimiento de las razones de deseabilidad de los fines a los que ha de encaminarse la educación (Gil Cantero, 1991).

En esta tarea las aportaciones de la Filosofía de la Educación son básicas para la Teoría de la Educación pero ésta no puede desentenderse de la reflexión sobre los fines. El teórico de la educación no es un mero técnico que operativiza secuencias de intervención de medios para fines "dados"; al contrario, es un especialista competente para precisar qué cambios son pedagógicos y cuáles pueden y deben lograrse en un determinado sujeto con fundamento de elección técnica.

Por otra parte, si es verdad que el concepto de educación es controvertido y escurridizo y se dan, con respecto a él, múltiples y diversas aproximaciones, una característica esencial, reconocida por todos, es la referencia a lo valioso. Por tanto la dimensión de los valores es una dimensión básica (Ibáñez-Martín, 1992; Medina, 1987) en la Teoría de la Educación que pretende explicar, comprender y normativizar la acción educativa. Una Teoría de la Educación implica siempre determinados presupuestos valorativos (Moore, 1980). Los objetivos a conseguir deben ser acordes con los valores y es también competencia y responsabilidad del teórico de la educación 
enjuiciar axiológicamente los patrones que se le proponen (Escámez, 1986). En definitiva, es básico saber "dónde se quiere ir", "qué es lo que se quiere conseguir" y "por qué", analizando la valiosidad de los patrones educativos.

- En otro orden de cosas, la educación, como hecho, como fenómeno y como proceso, se enmarca en una situación concreta -en unas coordenadas espacio/temporales-, configurada por diversos factores condicionantes (Castillejo, 1981): culturales, económicos y políticos. De estos factores derivan los valores vigentes en la sociedad que permiten concretar los fines educativos. La sociedad, además, utiliza diversos cauces y mecanismos para intervenir en el proceso educativo, que van desde el ordenamiento jurídico-político, que se concreta en política educativa, hasta el clima social, una vía difusa pero con importante incidencia (García Aretio, 2000). Se sirve, también, de otras vías más directas, las agencias e instituciones educativas, formales, no formales e informales, y de los profesionales de la educación. Por eso, la Teoría de la Educación debe incluir en su reflexión la consideración del marco sociocultural de la educación y de las profesiones $y$ agentes educativos.

- Una Teoría de la Educación debe partir del conocimiento disponible sobre el educando. El conocimiento actual sobre el hombre es fundamental: su educabilidad, los límites de la educación, las bases bioantropológicas de la misma, el funcionamiento del cerebro y de la mente humana, el hombre y su libertad, etc., son cuestiones de las que ha de partir la Teoría de la Educación y aquí las aportaciones de la Filosofía de la Educación, de la Antropología Pedagógica, de la Biología de la Educación, y del conjunto de las neurociencias son imprescindibles (Doval y Santos Rego, 1998; García Carrasco y García del Dujo, 2001).

- La Teoría del aprendizaje como base para la teoría de la enseñanza y, por tanto, también la teoría de la enseñanza son, también, cuestiones fundamentales, desde nuestro punto de vista, para la Teoría de la Educación. Si bien es cierto que la educación comporta algo más que aprendizaje, ya que no todo aprendizaje es educativo, la educación siempre conlleva e incorpora el aprendizaje y la enseñanza, aunque estemos de acuerdo con Altarejos (1991) en que la Teoría de la Educación no se puede basar sólo en la Psicología del aprendizaje y de la instrucción. Evidentemente, la lectura que se haga de las teorías del aprendizaje y de las teorías de la enseñanza ha de ser pedagógica, como hace Sarramona (2000), en su Teoría de la Educación. En este contexto, las aportaciones de la Filosofía, de la Psicología y especialmente de la Psicología cognitiva y de la Teoría del procesamiento de la información, se revelan fundamentales. 


\section{Los CONTENidos que SE tRABajan en TeORÍa de la EduCACión}

Hasta aquí hemos expuesto nuestra concepción inicial en torno al objeto de estudio, que contrastaremos en este apartado con lo que dicen y hacen los teóricos de la educación españoles. Lo haremos a partir de tres enfoques o niveles de concreción, ajustando progresivamente la "lupa" al tema que nos ocupa, analizando primero áreas de estudio e investigación recogidos de diversas fuentes, para pasar después a los manuales que se han ido editando sobre el tema en nuestro país y llegar, por fin, a los programas de la asignatura Teoría de la Educación.

\subsection{Una primera aproximación al tema}

Utilizaremos cuatro fuentes que se refieren a áreas de estudio o de investigación y a temáticas que ocupan a los profesores e investigadores del área de Teoría de la Educación, lo que supone que no son temáticas específicas y exclusivas de la disciplina:

1. El primer referente es el trabajo de investigación de Martínez y Buxarrais (1992), en que se recogen doce áreas de estudio e investigación en Teoría de la Educación, las siete primeras de carácter básico y las cinco siguientes de carácter aplicado.

2. El segundo es el trabajo de investigación de García Carrasco, García del Dujo, Barrón y González (1992), que analiza como indicadores los programas de las disciplinas docentes del área recopiladas de 11 universidades españolas, incluyendo asignaturas tan dispares como Pedagogía General, Filosofía de la Educación, Biología de la Educación, Fundamentos de Metodología, Teoría del Aprendizaje, etc., así hasta 33 disciplinas diferentes. Los autores encuentran nueve núcleos de trabajo investigador y académico analizando las denominaciones de las materias y los programas de las disciplinas.

3. El tercero son los Seminarios Interuniversitarios de Teoría de la Educación, celebrados desde 1982 (20 hasta el momento) y los Congresos Nacionales, celebrados desde 1986 ( 8 hasta el momento). Los primeros son foros de discusión exclusivos de los teóricos de la educación españoles que gozan de merecido prestigio y que han servido de modelo para otras áreas, y los segundos, más abiertos, permiten un mayor contacto con la sociedad - investigadores, personalidades relevantes en pedagogía, estudiantes, pedagogos, profesores, profesionales de la educación, etc.- - Hemos realizado una catalogación por áreas temáticas atendiendo fundamentalmente a los títulos de los mismos, que delimitan la temática general del Seminario o del Congreso. En unas ocasiones el rótulo permite su inclusión en una única área temática, en otras en más de un área. Una catalogación más estricta habría precisado de atención a todas las ponencias presentadas, una tarea excesiva 
para nosotros, en las actuales circunstancias, que, sin embargo consideramos necesario realizar en algún momento.

4. El cuarto son los artículos de las dos revistas del área (Teoría de la Educación. Revista Interuniversitaria; y Teoría de la Educación. Educación y Cultura en la Sociedad de la Información) ${ }^{7}$. Nosotros hemos analizado 91 artículos de la Revista Interuniversitaria desde el año 1991 —en que pasó a editarse en la Universidad de Salamanca- y los 14 de la revista electrónica ${ }^{8}$. La mayoría de los artículos se han ubicado en un campo temático determinado. En algún caso muy concreto - muy pocos- se ha ubicado en dos campos.

A continuación presentamos, integrada en un cuadro sinóptico (Tabla 1), la información procedente de las cuatro fuentes. En el caso de la columna correspondiente a los Seminarios y Congresos y también en la correspondiente a los artículos de las revistas, se ha incluido, entre paréntesis, el número de los mismos que aborda la temática.

7. Hay que tener presente, por un lado, que los profesores e investigadores que escriben en estas revistas no son sólo investigadores de la disciplina Teoría de la Educación -el abanico todavía se ha abierto más en la revista electrónica-, y, por otro, que los teóricos de la educación no sólo publicamos en "nuestras" revistas, sino que utilizamos también otros vehículos de difusión de nuestras investigaciones.

8. Nos ha llamado poderosamente la atención que haya poquísimos artículos referidos a trabajos empíricos y menos aún que recojan datos y evidencias de intervención pedagógica, un campo que entendemos también nuestro, de los teóricos de la educación, que pensamos que debería recibir acogida en nuestras revistas. 
TABLA 1

Áreas temáticas en Teoría de la Educación

\begin{tabular}{|c|c|c|c|}
\hline $\begin{array}{l}\text { Martínez y Buxarrais } \\
\text { (1992). Áreas }\end{array}$ & $\begin{array}{l}\text { García Carrasco y otros } \\
\text { (1992). Temas clave }\end{array}$ & $\begin{array}{c}\text { Seminarios y Congresos. } \\
\text { Temática }\end{array}$ & $\begin{array}{c}\text { Artículos de las revistas } \\
\text { de Teoría }\end{array}$ \\
\hline 1. Metodológica y conceptual & \multirow{2}{*}{$\begin{array}{l}\text { 1. Epistemología pedagógical } \\
\text { (Reflexión sobre temas educativos, } \\
\text { modos de racionalictad, tecnologial } \\
\text { educativa. planteamientos actuales } \\
\text { sobre la ciencia de la educación. } \\
\text { Intencionalidad: valores...) }\end{array}$} & $\begin{array}{c}\text { Cuestiones epistemológicas } \\
\text { y conceptuales }(5) \\
\text { Metodologías de } \\
\text { investigación (1) }\end{array}$ & $\begin{array}{l}\text { Aspectos epistemológicos } \\
\text { y conceptuales (21) } \\
\text { Metodologias de investigación } \\
\text { en Pedagogía y en Teoría } \\
\text { de la Educación (4) }\end{array}$ \\
\hline 2. Filosófica y axiológica & & 9 & Axiología (4) \\
\hline 3. Antropológica & $\begin{array}{l}\text { 2. Relaciones mente-cuerpo } \\
\text { 3. Procesos educacionales primarios }\end{array}$ & Antropología de la educación (1) & Antropología y educación (5) \\
\hline 4. Psicológica & $\begin{array}{l}\text { 4. Pedagogía de los procesos } \\
\text { cognitivos }\end{array}$ & 10 & $\begin{array}{c}\text { Teorias del aprendizaje } \\
\text { Pedagogía de los procesos } \\
\text { cognitivos. Pedagogía cognitiva (10) }\end{array}$ \\
\hline 5. Sociológica & & Pedagogía Social (1) & \\
\hline 6. Contextual & $\begin{array}{l}\text { 5. Educación y su entorno } \\
\text { (influencias socioambientales } \\
\text { en el comportamiento...) }\end{array}$ & & \\
\hline 7. Politica & $\begin{array}{l}\text { 6. El sistema de ensenanza. } \\
\text { Su estructura y planificación }\end{array}$ & $\begin{array}{l}\text { Política y planificación } \\
\text { de la educación (4) }\end{array}$ & Política y educación (5) \\
\hline 8. Educación moral & $\begin{array}{l}\text { 7. Pedagogia del } \\
\text { comportamiento moral }\end{array}$ & Educación moral (1) & Educación moral y civica (8) \\
\hline \multirow[t]{2}{*}{ 9. Animación sociocultural } & $\begin{array}{l}\text { 5. Educación y su entorno } \\
\text { (animación sociocultural...) }\end{array}$ & & $\begin{array}{l}\text { Animación sociocultural y } \\
\text { desarrollo comunitario }(5)\end{array}$ \\
\hline & $\begin{array}{l}\text { 5. Educación y su entorno } \\
\text { (educación ambiental...) }\end{array}$ & 11 & Educación ambiental (3) \\
\hline 10. Educación de adultos & & Formación permanente (1) & Educación de adultos (5) \\
\hline \multirow[t]{3}{*}{ 11. Pedagogía laboral } & & Educación y trabajo (2) & Pedagogia laboral (3) \\
\hline & $\begin{array}{c}\text { 8. Pedagogía del } \\
\text { comportamiento afectivo }\end{array}$ & 12 & \\
\hline & 9. Pedagogía de la comunicación & Comunicación y educación (2) & Comunicación y educación (9) \\
\hline \multirow[t]{2}{*}{ 12. Profesiones educativas } & & La profesión docente (1) & $\begin{array}{l}\text { La profesión docente. } \\
\text { Deontología profesional. } \\
\text { Formación de profesores (9) }\end{array}$ \\
\hline & & Curriculum (2) & Curriculum (4) \\
\hline \multirow[t]{8}{*}{$\begin{array}{c}\text { 8. Educación moral } \\
\text { (educación intercultural) }\end{array}$} & & Educación Intercultural (2) & $\begin{array}{c}\text { Educación Intercultural/ } \\
\text { multicultural }(6)\end{array}$ \\
\hline & & Educación no formal (2) & Educación no formal (4) \\
\hline & & $\begin{array}{c}\text { Evaluación (políticas educativas) } \\
\text { (1) }\end{array}$ & $\begin{array}{l}\text { Evaluación (del sistema, de } \\
\text { la universidad, de políticas) (6) }\end{array}$ \\
\hline & & Educación universitaria (1) & Educación universitaria (3) \\
\hline & & $\begin{array}{l}\text { Cambio y educación para } \\
\text { el cambio (2) }\end{array}$ & \\
\hline & & 15 & Educación familiar (1) \\
\hline & & Educación y calidad de vida (1) & \\
\hline & & Conflicto, violencia y educación (1) & \\
\hline
\end{tabular}

9. Aunque no existan Seminarios o Congresos dedicados íntegramente al tema, éste se ha hecho presente en muchos de ellos en varias ponencias: así, en el IV Seminario, en el V, en el XIII, etc.

10. Ocurre lo mismo que en el caso anterior, y así la temática se ha tratado al menos en ponencias en el IV Seminario y en el XV.

11. También la educación ambiental ha sido tratada en ponencias, al menos en el II Seminario y en el VII.

12. También la educación afectiva ha sido tratada en ponencias, al menos en el XIII Seminario.

13. La temática reaparece en ponencias en el $7^{\circ}$ Congreso. 
Aparecen algunas temáticas fundamentales que se repiten analizando las cuatro fuentes mencionadas y con mayor presencia en los Seminarios y Congresos y en los artículos de las revistas y que ordenadas son las que siguen:

1. Cuestiones epistemológicas y conceptuales referidas a la Pedagogía y a la Teoría de la Educación, con especial hincapié en la tecnología de la educación.

2. Cuestiones de antropología de la educación

3. Procesos cognitivos y educación. Pedagogía cognitiva.

4. Política y planificación de la educación.

5. Educación moral.

6. Profesiones educativas. Formación de profesores. Deontología profesional.

7. Comunicación y educación.

8. Educación Intercultural.

9. Metodologías de investigación.

10. Educación de adultos. Formación Permanente.

11. Pedagogía laboral.

12. Animación sociocultural.

13. Educación no formal.

14. Evaluación.

15. Currículum.

16. Filosofía y axiología.

17. Etc.

Queremos insistir, una vez más, en que se trata de temáticas que ocupan a los profesores e investigadores del "área" (o subárea), bien a nivel de reflexión y de investigación bien a nivel de docencia, y queremos remarcar que en el área convergen diversas disciplinas docentes, lo que explica la diversidad de temas y la riqueza de contenidos de los mismos. De hecho, algunas de las áreas temáticas abordadas tienen tal entidad que configuran disciplinas científicas y docentes: Pedagogía Social, Educación Moral, Antropología de la Educación, Política Educativa, Formación Permanente...

En todo caso, desde este primer enfoque del tema y con esta primera aproximación de tipo más general, se confirman algunas temáticas básicas que coinciden con las que nosotros apuntamos como objeto de la Teoría de la Educación, disciplina científica, que deberían ser recogidas, por tanto, en la misma, precisamente por ese carácter integrador, de fundamentación y de síntesis que le atribuimos. Entendemos que se trata de temas que encuentran un "espacio" idóneo de ubicación en la Teoría de la Educación, que les confiere sentido y coherencia desde la perspectiva fundamentante e integradora antes aludida, aunque algunos de ellos también lo encuentren en otras disciplinas del área: así, cuestiones epistemológicas

14. Los autores incluyen la educación intercultural como tópico o tema dentro de la educación moral. 15. Se le dedica, al menos, una ponencia en el VII Seminario. 
y conceptuales, metodología de investigación, elementos de antropología de la educación, cuestiones axiológicas y teleológicas, procesos cognitivos, aprendizaje y educación, comunicación y educación, profesión docente, etc. También aparecen otras temáticas relevantes en el ámbito de la Teoría de la Educación que nosotros no contemplamos en su momento - si bien es cierto que algunas de ellas se incluyen en el proceso educativo, que para nosotros es el objeto fundamental de reflexión e indagación en la disciplina - y que tienen pleno sentido en la Teoría de la Educación que propugnamos: así, nuevas tecnologías de la información y la comunicación y educación, aspectos curriculares, evaluación, etc.

Hemos detectado que, de un claro énfasis inicial puesto en cuestiones conceptuales y epistemológicas, evidente en los primeros seminarios ${ }^{16}$ y en las publicaciones de ellos derivadas, y también en los artículos de los primeros números de la revista "de papel” — que es lógico cuando se pretende clarificar y consolidar una disciplina científica y un área de conocimiento-, se ha ido pasando a un interés creciente por temas sociales relevantes (educación intercultural, educación y calidad de vida, cambio y educación para el cambio, educación y violencia, etc.) sobre los que los teóricos de la educación debemos reflexionar y aportar ideas. Así y todo, los temas epistemológicos siguen ocupando un lugar fundamental en la reflexión de los teóricos de la educación (basta con consultar nuestros seminarios y revistas así como los manuales y programas a los que luego hacemos mención), sin dejar de lado otros temas que pueden considerarse fundamentales: así, antropología de la educación, comunicación y educación, pedagogía y procesos cognitivos, educación moral, etc.

Se evidencia, por tanto, un núcleo de temáticas básicas e irrenunciables para la Teoría de la Educación.

Se trata, no obstante, de una primera aproximación al problema que nos ocupa, que debe ser matizada con las aportaciones de otras fuentes, ya que las consultadas hasta aquí recogen temas del área, no exclusivamente de la disciplina.

\subsection{Una segunda aproximación al tema}

Procederemos, en este caso, a analizar los manuales de Teoría de la Educación que, si bien en algunos casos se han elaborado pensando en una disciplina docente - asignatura de plan de estudios_- en la mayoría lo han sido más bien, o también, desde la perspectiva de la construcción de la disciplina científica. Al menos ésa es nuestra percepción. Será, por tanto, ésta una fuente fundamental de indagación para precisar los contenidos de la disciplina.

16. El primer Seminario (1982) versó sobre «La Teoría de la Educación: líneas de investigación y ámbitos de actuación", el segundo (1983) sobre "El problema de la educación", y el cuarto (1985) sobre "Tecnología de la acción educativa". 
Realizamos, a continuación, un breve análisis de los temas que tratan dichos manuales ${ }^{17}$. Para ello se han consultado los de los siguientes autores: Colom y Núñez Cubero (2001), Sarramona (2000), Medina, Rodríguez y García (2000), Aznar (1999), García Carrasco y García del Dujo (1996 y 2001) ${ }^{18}$, Castillejo, Vázquez, Colom y Sarramona (1994), y Ferrero (1994), editados con posterioridad a la implantación de los nuevos planes de estudios o coetáneos de la misma. También se ha hecho lo propio con obras de Teoría de la Educación anteriores de los siguientes autores: Quintana (1988), Touriñán (1987), Puig (1986), Castillejo, Cervera, Colom, Escámez, Esteve, García Carrasco, Marín, Sanvicens, Sarramona y Vázquez (1983), Fermoso (1982), Castillejo, Escámez y Marín (1981), Nassif (1980), Capitán, (1977), y Tusquets (1972).

El análisis se ha llevado a cabo rastreando la presencia de temas fundamentales. Hemos partido, para ello, del estudio previo referido a temáticas que ocupaban la reflexión e investigación de los teóricos de la educación, cuyos resultados reflejamos antes. A partir de este listado inicial se han añadido los nuevos temas que han aparecido.

Los temas de los que hemos partido y sus descriptores son los siguientes:

1. Cuestiones conceptuales: Concepto de educación. El fenómeno educativo. Educación como proceso y como resultado. La acción educativa...

2. Cuestiones epistemológicas: El saber científico de la educación. Estatuto epistemológico de la Teoría de la Educación. Teoría de la Educación y CC. de la Educación. Tecnología educativa. Integración teoría-praxis...

3. Cuestiones metodológicas: Paradigmas de investigación educativa. Metodologías de investigación en Pedagogía y en Teoría de la Educación...

4. Axiología/Valores/Fines: Axiología educativa. El modelo de hombre. Los valores. Fines y valores. Finalidades de la educación...

5. El sujeto de la educación. Bases antropológicas: La estructura psicobiológica del ser humano y la educación. El hombre, sujeto de la educación. El hombre ser educable. Educabilidad. Desarrollo cognitivo y social..

17. Hemos seleccionado todos los manuales de los que teníamos referencia, al menos de la última época, que aparecían en las bases de datos. Es posible que no se haya considerado algún manual importante de la época que antecede a la reforma de los planes de estudios. Si fuera así. no se trata de un olvido "premeditado". En todo caso, son todos los que están aunque no estén todos los que son.

18. Los autores editaron en 1996 un primer volumen titulado Teoría de la Educación I. Educación y acción pedagógica y en 2001 un segundo volumen titulado Teoría de la Educación II. Procesos primarios de formación del pensamiento y la acción. Anuncian, en este segundo volumen, un tercero, que completará la obra. En el primero abordaron, fundamentalmente, cuestiones conceptuales y epistemológicas (como dicen los autores: ¿Qué cosa es la educación y cómo reflexionar sobre ella?), en el segundo cuestiones antropológicas que posibilitan y exigen la educación y cuestiones referidas al entorno en que ésta se realiza (¿Cómo somos para poder ser educables y qué acontece al educarnos?), y en el tercero se proponen abordar las cuestiones referidas a las estrategias y contenidos de las acciones de formación (¿Qué es importante hacer para educarse?). 
6. Teoría de los procesos educativos: La educación como proceso. Procesos de acción educativa. Procesos de planificación. Decisiones pedagógicas. Procesos de aprendizaje. Procesos de construcción personal: Procesos de evaluación. Tecnología de los procesos de intervención... ${ }^{19}$.

7. Factores condicionantes y socioculturales: La educación como fenómeno social. Interacción sociedad-educación. Factores condicionantes...

8. Instituciones y agencias educativas: La familia como agencia educativa. La escuela. El sistema educativo reglado. Instituciones de educación no formal...

9. Dimensiones de la educación: Dimensión afectiva. Dimensión estética. Dimensión física. Dimensión moral. Dimensión social. Dimensión intelectual...

10. Educación formal, no formal e informal: Concepto. Aplicaciones...

11. La educación como comunicación. Nuevas tecnologías de la información y la comunicación: Concepto de comunicación. La educación como proceso y como sistema de comunicación. Nuevas tecnologías de la información y la comunicación y educación...

12. El currículum: Concepto de currículum. Fundamentos. El currículum escolar. Los contenidos. Tipos de contenidos...

13. Teorías y modelos de aprendizaje: Teorías del aprendizaje. Implicaciones pedagógicas de los modelos de aprendizaje. Modelos pedagógicos de aprendizaje...

14. La profesión educativa: El educador. El profesor. Función docente. La profesión pedagógica, etc.

Los resultados se recogen en la Tabla 2. En las cabeceras de las columnas aparecen los manuales. En las de las filas las temáticas. Los manuales se presentan ordenados por fecha de publicación. Los primeros han sido publicados, como dijimos con la reforma de los planes o después de ella. Los segundos, anteriores, aparecen separados de los primeros con una línea más gruesa. La presencia de la temática en el manual correspondiente se marca con tono oscuro.

Los temas marcados en los diferentes manuales tienen una presencia explícita en los mismos. Es cierto que no en todos tienen el mismo peso: Así, la teoría de los procesos educativos ocupa en Aznar (1999) casi tres cuartas partes de la obra, que es voluminosa, y en Colom y Núñez Cubero (2001) una parte sustantiva; en otros manuales se le dedica sólo un capítulo. La antropología y educación; bases antropológicas, otro de los temas que consideramos nucleares, en Medina, Rodríguez y García (2000) ocupa una sección, cinco temas y casi 100 páginas, mientras que en el volumen de 1996 del manual de García Carrasco y García del Dujo no aparece como tal elemento diferenciado, al contrario de lo que ocurre en el volumen de 2001, que se dedica en buena parte al tema antropológico. En el manual de Aznar (1999) sólo ocupa un breve subapartado en que se aborda el tema de la educabilidad.

19. Algunos de los temas que aparecen como tales podrían incluirse aquí. Así, currículum, axiología, procesos de comunicación y educación, etc. Sin embargo, dada su entidad, los hemos incluido como temas con autonomía. 


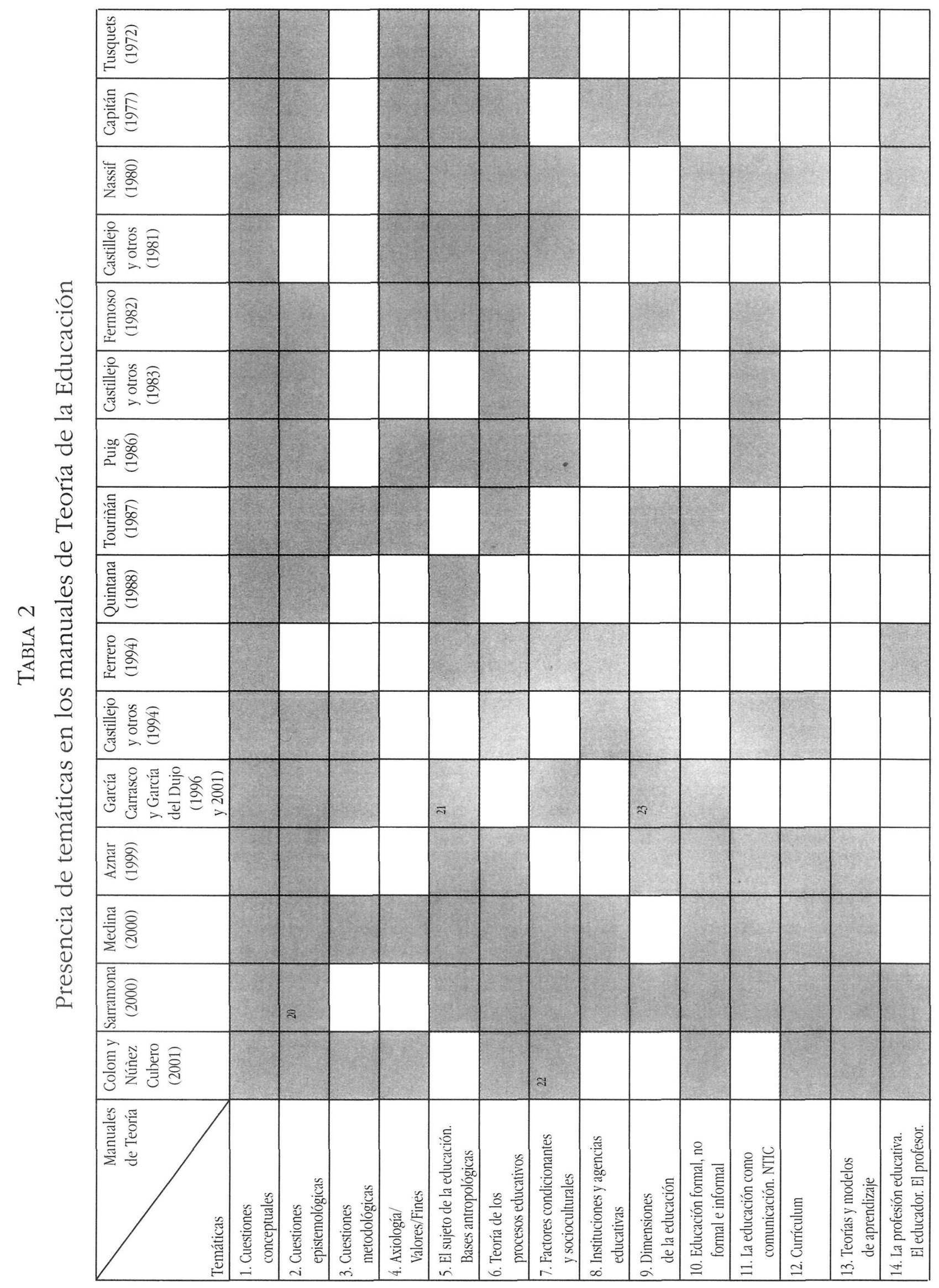


Obsérvese que, en los siete manuales más recientes, hay una serie de temáticas que tienen una presencia mayor. La relación ordenada es la siguiente:

1. Con presencia en los siete manuales:

Cuestiones conceptuales.

2. En seis manuales:

Cuestiones epistemológicas.

Teoria de los procesos educativos.

3. En cinco manuales:

El sujeto de la educación/Bases antropológicas.

Educación formal, no formal e informal.

Curriculum.

Factores condicionantes y socioculturales.

4. En cuatro manuales:

Cuestiones metodológicas.

Dimensiones de la educación.

Instituciones y agencias educativas.

La educación como comunicación/Nuevas Tecnologías.

Teorias y modelos de aprendizaje.

5. En tres manuales:

La profesión educativa. El educador.

6. En dos manuales:

Axiología. Fines y valores.

Hay una coincidencia sustantiva entre manuales recientes y manuales más antiguos en la mayoría de los temas fundamentales, lo que supone una línea clara de continuidad y la existencia de un corpus básico que sería insoslayable en la disciplina y que se sostiene a lo largo del tiempo. Así, todos ellos abordan cuestiones conceptuales, y casi todos cuestiones epistemológicas (ocho de los nueve más antiguos y seis de los siete más recientes). Otros temas compartidos con altas frecuencias son teoría de los procesos educativos (presente en siete de los nueve más antiguos y en seis de los siete más recientes), sujeto de la educación; bases antropológicas (en siete de los nueve más antiguos y cinco de los siete más recientes), y educación como comunicación (en cuatro de los más antiguos y en cuatro de los más recientes). Encontramos después otras temáticas presentes en ambos tipos de

20. Sólo las toca explícitamente en la introducción.

21. En el volumen de 2001 se profundiza en elementos como la identidad e imagen del cuerpo, el órgano de la mente, plasticidad y educabilidad, actividad mental, etc.

22. Se incluye alguna referencia en el capítulo dedicado a ética y valores

23. Se dedica un capítulo entero a la dimensión afectiva en el mismo volumen de 2001. 
manuales, pero con más peso en los actuales: así factores condicionantes y socioculturales (con presencia en cinco de los siete manuales más recientes pero sólo en cuatro de los nueve más antiguos), educación formal, no formal e informal (en cinco de los siete manuales más recientes y sólo en dos de los más antiguos), dimensiones de la educación (en cuatro de los manuales más recientes pero sólo en tres de los manuales más antiguos), cuestiones metodológicas (en cuatro de los siete manuales más recientes y sólo en uno de los nueve más antiguos), currículum (en cinco de los siete más recientes y sólo en uno de los más antiguos), instituciones y agencias educativas (en cuatro de los manuales más recientes y sólo en uno de los más antiguos), la profesión educativa (en tres de los manuales más recientes y sólo en dos de los más antiguos). Por fin, se constata una reducción llamativa en el tema de axiología, con respecto a manuales anteriores de Teoría (presente en siete de los manuales más antiguos pero sólo en dos de los más recientes), y la aparición de una temática nueva, la referida a teorias y modelos de aprendizaje, no recogida en los manuales más antiguos.

En definitiva, se mantienen con fuerza algunas de las temáticas que pueden considerarse tradicionales de la Teoría de la Educación -irrenunciables, en último término- (epistemologia, elementos conceptuales, bases antropológicas, teoría de los procesos educativos...), cobran fuerza otras que tenían menos peso en los manuales anteriores a la reforma de los planes de estudios (factores condicionantes y socioculturales, instituciones y agencias educativas, profesiones educativas -obsérvese que se trata de temáticas vinculadas al marco sociocultural de la educación, que incrementan su presencia-, curriculum, comunicación y educación, cuestiones metodológicas, etc.), sufren reducción sustancial otras (axiología...), lo que está en franca discrepancia con nuestra posición de defensa sin ambages de la presencia de esta temática en Teoría de la Educación (punto 2 de este artículo) y aparece una nueva, teorias y modelos de aprendizaje, no recogida en los manuales anteriores a la reforma, lo que es coherente con los hallazgos del apartado anterior en que se contemplaba el área psicológica/teorías del aprendizaje/pedagogía de los procesos cognitivos como área temática importante. Todo ello parece apuntar a una presencia progresivamente más firme de contenidos con carga sociológica y psicológica (psicología del aprendizaje) que complementan los de origen más filosófico-antropológico.

\subsection{Una tercera aproximación al tema}

La Teoría de la Educación, disciplina pedagógica sustantiva o disciplina científica, se subdivide en disciplinas docentes (asignaturas de plan de estudios) (Medina, 2000a; Tourinaán, 1987), que responden a la tradición docente e investigadora de las diferentes universidades, a la producción de conocimiento y también a razones pragmáticas. Además de analizar los manuales de Teoría de la Educación, hicimos lo propio con los programas de la asignatura Teoría de la Educación de las 
diferentes universidades españolas, lo que nos permitiría determinar los temas que abordan los profesores en la disciplina docente, al reflejar los programas, con bastante fidelidad, los contenidos impartidos. Ello nos daría pie a completar la información recogida de las otras fuentes, siendo conscientes, por supuesto, de que en este caso se trata de una asignatura de plan de estudios que, en todo caso, habría que interpretar como un "subconjunto" de la disciplina científica - no se puede integrar en una asignatura todo el corpus científico de la misma-.

Llevamos a cabo el análisis sobre 18 programas - recientes, la mayoría impartidos en 2000/2001 - de la asignatura "Teoría de la Educación" que fue posible conseguir de 15 universidades españolas. En tres de las universidades se dispuso de dos programas distintos, bien por ser impartida la materia por diferentes profesores que no compartían completamente el programa, bien por disponer de programas del curso actual y de algún curso anterior. En estos casos, en el recuadro correspondiente a la universidad aparece también, entre paréntesis, un número que distingue los programas analizados. Somos conscientes de que la interpretación de los epígrafes de los programas es personal. En la mayoría de los casos entendemos que es inequívoca por la univocidad de los términos. En otros podrían caber diferentes lecturas de los temas y epígrafes que los reflejan. Además, el peso dado a las diferentes temáticas también varía, oscilando de un núcleo temático, que comporta diferentes temas y unidades, a un tema del programa o incluso - en contadas ocasiones- a un epígrafe de un tema. A eso se añade que unos programas son más pormenorizados que otros.

En la tabla siguiente (Tabla 3) se recogen las temáticas que venimos contemplando, y las hemos presentado siguiendo el mismo orden anteriormente fijado. Se han añadido temáticas nuevas que aparecen en los programas, no presentes en los análisis anteriores. En cada uno de los casos, entre paréntesis, aparece el número correspondiente a la frecuencia de aparición, es decir, al número de programas en los que se incluye. 
TABLA 3

Presencia de temáticas en los programas de Teoría de la Educación

\begin{tabular}{|c|c|c|c|c|c|c|c|c|c|c|c|c|c|c|c|c|c|c|}
\hline Temáticas & Granada & $\begin{array}{c}\text { Murcia } \\
\text { Barcelona } \\
\text { (1) }\end{array}$ & $\begin{array}{c}\text { Autónoma } \\
\text { Barcelona } \\
\text { (2) }\end{array}$ & Autónoma & Salamanca & $\begin{array}{l}\text { UNED } \\
\text { laguna }\end{array}$ & $\begin{array}{l}\mathrm{La} \\
(1)\end{array}$ & $\begin{array}{l}\text { Santiago } \\
\text { (2) }\end{array}$ & Santiago & $\begin{array}{l}\text { Comillas } \\
\text { (1) }\end{array}$ & $\begin{array}{l}\text { Valencia } \\
\text { (2) }\end{array}$ & Valencia & Naviarra & Oviedo & Sevilla & Málaga & Baleares & Barcelona \\
\hline \multicolumn{19}{|l|}{ 1. Cuestiones conceptuales (18) } \\
\hline \multicolumn{19}{|l|}{ 2. Curestiones episitemológicas (18) } \\
\hline \multicolumn{19}{|l|}{ 3. Cuestiones metodologogicas (5) } \\
\hline \multicolumn{19}{|l|}{ 4. Axiología. Valores. Fines (11) } \\
\hline \multicolumn{19}{|l|}{ 5. Suijeto educ. Bases antropol. (13) } \\
\hline \multicolumn{19}{|l|}{ 6. Teoría de los procesos educ. (10) } \\
\hline \multicolumn{19}{|l|}{$\begin{array}{l}\text { 7. Factores condicionantes y } \\
\text { contextuales socioculturiales (6) }\end{array}$} \\
\hline \multicolumn{19}{|l|}{ 8. Instituciones y agencias educ. (10) } \\
\hline \multicolumn{19}{|l|}{ 9. Dimensiones de la educación (9) } \\
\hline \multicolumn{19}{|l|}{ 10. Educ. no formal, formal.... (12) } \\
\hline \multicolumn{19}{|l|}{ 11. La educ. como comunic. TIC (11) } \\
\hline \multicolumn{19}{|l|}{ 12. Currículum (8) } \\
\hline \multicolumn{19}{|l|}{ 13. Teorías y modelos de aprend. (6) } \\
\hline \multicolumn{19}{|l|}{ 14. La professión educativia (10) } \\
\hline \multicolumn{19}{|l|}{ 15. Educ. permanente y de adultos (5) } \\
\hline \multicolumn{19}{|l|}{ 16. Educación como sistema (3) } \\
\hline \multicolumn{19}{|l|}{ 17. Calidad y particip. en educ. (3) } \\
\hline \multicolumn{19}{|l|}{ 18. Educación a distancia (2) } \\
\hline \multicolumn{19}{|l|}{ 19. Atención a la diversidad (3) } \\
\hline \multicolumn{19}{|l|}{ 20. El sistema educativo (4) } \\
\hline \multicolumn{19}{|l|}{ 21. Sistema educ-sistema laboral (2) } \\
\hline \multicolumn{19}{|l|}{ 22. Educación y Europa (2) } \\
\hline \multicolumn{19}{|l|}{ 23. Procesos de reforma educ. (1) } \\
\hline \multicolumn{19}{|l|}{ 24. Educacaín y siglo XXI (1) } \\
\hline 25. Tendenciass y corrientes petag. (1) & & & & & & & & & & & & & & & & & & \\
\hline
\end{tabular}

Nota: en la Universidad de Barcelona se imparten dos disciplinas obligatorias de 6 créditos: Teoría de la Educación I y Teoría de la Educación II, ambas de 6 créditos. También se imparte otra disciplina obligatoria titulada Paradigmas de la Teoría de la Educación, de 4,5 créditos. Por coherencia con el análisis que venimos realizando de programas de las diferentes universidades, sólo hemos contemplado aquí los contenidos de las disciplinas rotuladas exclusivamente como Teoría de la Educación. 
La relación ordenada de temáticas, en función de la mayor o menor presencia, es la siguiente:

1. Con presencia en los dieciocho programas:

Cuestiones conceptuales.

Cuestiones epistemológicas.

2. En trece programas:

El sujeto de la educación. Bases antropológicas.

3. En doce programas:

Educación formal, no formal e informal.

4. En once programas:

Axiologia/Valores/Fines.

Educación y comunicación/Nuevas tecnologias de la información

y la comunicación.

5 En diez programas:

Teoría de los procesos educativos.

Instituciones y agencias educativas.

La profesión educativa/El educador/El profesor.

6. En nueve programas:

Dimensiones de la educación.

7. En ocho programas:

Curriculum.

8. En seis programas:

Factores condicionantes y socioculturales.

Teorias y modelos de aprendizaje.

9. En cinco programas;

Cuestiones metodológicas.

Educación permanente $y$ de adultos.

10. En cuatro programas:

El sistema educativo.

11. En tres programas;

La educación como sistema.

Calidad y participación en la educación.

Atención a la diversidad.

12. En dos programas;

Educación a distancia.

Sistema educativo-sistema laboral.

Educación y Europa. 


\section{En un programa.}

Educación y siglo XXI.

Procesos de reforma educativa.

Tendencias y corrientes pedagógicas.

Se confirman una serie de temáticas fundamentales que han ido apareciendo en los análisis anteriores. Así, cuestiones conceptuales y cuestiones epistemológicas (presentes en los dieciocho programas), el sujeto de la educación/bases antropológicas (en trece de los dieciocho programas), educación formal, no formal e infor mal (en doce), axiologialvalores/fines, educación y comunicación/nuevas tecnologias (presentes en once programas), teoria de los procesos educativos, instituciones y agencias educativas, la profesión educativa (presentes en diez programas) y dimensiones de la educación (en nueve programas). Otros temas con presencia aceptable, pero en menor número de programas, son curriculum (en ocho programas), factores condicionantes y socioculturales, teorias y modelos de aprendizaje (en seis), cuestiones metodológicas y educación permanente de adultos (en cinco). Por fin, con presencia en cuatro programas o menos tenemos el sistema educativo, la educación como sistema, calidad y participación en la educación, atención a la diversidad, educación a distancia, sistema educativo-sistema laboral, educación y Europa, educación y siglo XXI, procesos de reforma educativa, $y$ tendencias y corrientes pedagógicas (las tres últimas con presencia en un solo programa).

Todo ello supone que el corpus básico de la disciplina científica, que se ha ido perfilando a partir de las dos aproximaciones previas, se sostiene en los programas de la asignatura del mismo nombre. Queremos llamar la atención sobre el hecho de que la temática relativa a axiologia/valores/fines, que tenía una presencia mínima en los manuales analizados en el apartado anterior, gana presencia en los programas de los profesores, al encontrarse en once de los dieciocho programas. Por otra parte, aparecen nuevos temas que de momento, tiene una presencia mucho menor en los programas (calidady participación en la educación, atención a la diversidad, sistema educativo-sistema laboral, etc.). Estas nuevas temáticas tienen que ver con intereses profesionales y de investigación de los profesores que los imparten: vgr. educación permanente (Santiago, 1), educación a distancia (UNED), con el intento de abrir la Teoría de la Educación a problemas actuales relevantes a nivel social: educación y Europa, calidad y participación en la educación, atención a la diversidad, etc., y también con la tradición y sensibilidad de las diferentes universidades y de sus profesores. 


\section{Conclusiones}

Partimos en este artículo de una propuesta personal en torno a lo que entendíamos que era el objeto de la Teoría de la Educación como disciplina científica. Esta propuesta fue contrastada con las aportaciones de diversas fuentes relativas a contenidos trabajados: los provenientes del análisis de las temáticas fundamentales abordadas por la Teoría de la Educación como área de conocimiento, los extraídos de los manuales de la disciplina y los propuestos en los programas de la asignatura. Encontramos una notable coherencia entre lo que nosotros proponíamos, lo que trabajan los teóricos de la educación a nivel de áreas de estudio e investigación, lo que recogen los manuales que publican los colegas y lo que los profesores enseñan -o explicitan que enseñan a través de sus programas docentes-.

A partir de todo ello, nuestra propuesta de temáticas fundamentales, objeto de reflexión y de investigación científica para los que trabajamos en la disciplina, sería la siguiente:

1. Cuestiones conceptuales básicas (La educación como hecho, como fenómeno, como acción, como sistema y como proceso. La educación y su red nomológica. La acción educativa. La acción pedagógica. Educación formal, no formal e informal. Dimensiones de la educación —intelectual, física y psicomotriz, afectiva, estética, moral, social, etc.-...).

2. Cuestiones epistemológicas (El saber científico de la educación. La evolución de la reflexión en torno a la educación en la historia. Modos de racionalidad para afrontar el problema. La pedagogía como ciencia y tecnología de la educación. El estatuto epistemológico de la Teoría de la Educación. Teoría de la Educación, Pedagogía y Ciencias de la Educación. Tecnología educativa. Integración teoría-praxis en educación...).

3. Cuestiones metodológicas (Paradigmas de investigación educativa. Metodologías de investigación en Pedagogía y en Teoría de la Educación. La investigación en Teoría de la Educación...).

4 El sujeto de la educación. Bases antropológicas (La estructura psicobiológica del ser humano y la educación. El hombre sujeto de la educación. El hombre, ser educable. Plasticidad y educabilidad. Cerebro y mente. Herencia-medio. Desarrollo cognitivo y social. Desarrollo moral. El autoconcepto como dimensión personal. Aprendizaje adulto...).

5. Elementos y agentes intervinientes en el proceso educativo (El marco sociocultural de la educación. Factores condicionantes. Instituciones y agencias educativas. La familia. La escuela. Las instituciones educativas no formales. La "ciudad educativa". Los profesionales de la educación...).

6. Axiología/Fines/Valores (El marco teleológico y axiológico de la educación. Los valores, fundamento de la educación y de sus fines. Los objetivos educativos. Criterios de selección de fines y objetivos. Taxonomías...).

7. Teoría de los procesos educativos (La educación como proceso. Modelos conceptuales del proceso educativo. Procesos de diseño o planificación. Procesos 
de acción educativa — procesos de comunicación educativa, metodologías y estrategias de intervención, medios educativos directos y medios indirectos o facilitadores...-. Procesos de aprendizaje - Procesos de aprendizaje de conocimientos, procedimientos, valores y normas, procesos de génesis de consistencias (aptitudes, hábitos, actitudes, estilos cognitivos...), procesos estratégicos y metacognitivos, procesos de autocontrol y autonomía, procesos creativos, procesos emotivo-afectivos...-. Procesos de evaluación... $)^{24}$.

8. El currículo (Fundamentos y fuentes. Componentes del currículo. Los contenidos educativos. Criterios de selección de contenidos. Diseño y desarrollo curricular. La transversalidad en la educación -educación moral, educación ambiental, educación para la salud, educación para la paz, etc.-....).

9. La educación como comunicación (La comunicación humana. Comunicación, información y educación. La educación como proceso y sistema de comunicación. Comunicación y construcción personal. Nuevas tecnologías de la información y la comunicación y educación. Internet y su uso educativo...).

10. Teorías y modelos de aprendizaje (Teorías del aprendizaje. Implicaciones pedagógicas. Modelos pedagógicos de aprendizaje...).

Entendemos que este planteamiento es lo suficientemente amplio y comprensivo y que en él tienen cabida líneas de investigación y trabajos tanto de carácter básico como aplicado ${ }^{25}$ que estamos desarrollando los que nos decimos teóricos de la educación, referidos a temas y problemas educativos relevantes — también a nivel social-, que son coherentes con las temáticas apuntadas y que los investigadores consideramos propios de la Teoría de la Educación: así, educación moral, educación para la ciudadanía, educación ambiental, educación y desarrollo sostenible, educación para la salud, prevención de la drogadicción, valores y educación, enseñanza de actitudes y valores, las normas y la intervención educativa, estilos cognitivos e intervención pedagógica, educación intercultural, enseñanza de estrategias de aprendizaje, el autoconcepto y su mejora, comunicación y educación,

24. Como hicimos constar en su momento, las tres temáticas que siguen, e incluso la que precede a ésta, podrían considerarse parte de la misma, parte del proceso educativo, porque lo son. Sin embargo, tienen tal entidad que las hemos considerado como temáticas dignas de ser abordadas como tales.

25. Desde nuestro punto de vista son perfectamente compatibles los trabajos de carácter básico, de amplia tradición en el área, con los que un sector de teóricos de la educación se siente muy identificado, con otros de carácter aplicado, incluyendo trabajos empíricos y programas de intervención con diseños cuantitativos, precisos en ocasiones, con los que otro sector de teóricos de la educación también lo está, a los que progresivamente deberían abrirse nuestras dos revistas, tal como nosotros lo vemos. No en vano, el énfasis en los procesos educativos ha sido también tradicional en el área. Tan es así que hace unos años se solicitó al Consejo de Universidades el cambio de denominación del área, que hubiera pasado a denominarse "Pedagogía y procesos educativos", en lugar de "Teoría de la Educación”. Tal solicitud, que sepamos, no ha sido satisfecha. También la orientación normativo-tecnológica, muy importante en el área, ha inducido a un importante sector de investigadores del área de diferentes universidades españolas a trabajar en intervención educativa. 
nuevas tecnologías de la información y la comunicación y educación, Internet y su uso educativo, educación de adultos, educación no formal, formación de profesores, etc., por dejar constancia de algunas líneas de investigación de las que tenemos conocimiento en las que se está trabajando.

Ésta es nuestra propuesta, una propuesta para debate, discutible por tanto, y mejorable con toda seguridad.

\section{BIBLIOGRAFIA}

Altarejos, F. (1991) La acción educativa: enseñanza y formación, en Altarejos, F.; Bouché, J.; escámez, J.; Fullat; O.; Fermoso, P.; Gervilla, E.; Gil, R.; Ibáñez Martín, J. A.; Marín, R.; Pérez, P. M. a y SACRISTÁn, D. Filosofía de la Educación Hoy. Conceptos. Autores. Temas. Madrid, Dykinson, 603-619.

Angulo, J. F. y Blanco, N. (coords.) (1994) Teoria y desarrollo del curriculum. Málaga, Aljibe. ARISTÓteles (1985) Ética a Nicómaco. Madrid, Centro de Estudios Constitucionales. Edición bilingüe y traducción de María Araújo y Julián Marías. $4^{\underline{a}}$ ed.

Aznar, P. (coord.) (1999) Teoría de la educación. Un enfoque constructivista. Valencia, Tirant lo Blanch.

AZnar, P. y Requejo, A. (1993) La investigación etnográfica en la educación no formal, en Núñez Cubero, L. (ed.). Metodologías de investigación en la educación no formal. Aportaciones teóricas. Sevilla, PreuSpínola, 135-186.

BARrio, J. M. y Ruiz, M. (1992) La Filosofía de la Educación y la realidad pedagógica alemana en la segunda mitad del siglo xx, en Bárcena, F.; Gil, F.; Ibáñez-Martín, J. A.; Jover, G.; Ruiz CORBElla, M. y SACRISTÁn, D. La Filosofía de la Educación en Europa. Madrid, Dykinson, 25-38.

Bunge, M. (1980) Epistemología. Barcelona, Ariel.

- (1981) La ciencia, su método y su filosofía. Buenos Aires, Siglo XXI.

CAMPBElL, D. T. y STANLeY, J. (1979) Diseños experimentales y cuasiexperimentales en la investigación social. Buenos Aires, Amorrortu.

CAPITÁn, A. (1977) Teoría de la Educación. Granada, Universidad de Granada. ICE División de Orientación.

Castillejo, J. L. (1981) El marco socio-cultural de la educación, en Castillejo, J. L.; Escámez, J. y Marín, R. Teoría de la Educación. Madrid, Anaya 2, 37-64.

- (1987) Pedagogía Tecnológica. Barcelona, Ceac.

Castillejo, J. L.; Cervera, A.; Colom, A. J.; Escámez, J.; Esteve, J. M.; García Carrasco, J.; Marín, R.; Sanvicens, A; Sarramona, J. y Vázquez, G. (1983) Teoría de la Educación I. (El problema de la educación). Murcia, Límites.

Castillejo, J. L. y Colom, A. J. (1987) Pedagogia Sistémica. Barcelona, Ceac.

CASTIllejo, J. L.; Escámez, J. y Marín, R. (1981) Teoría de la Educación. Madrid, Anaya 2.

Castillejo, J. l.; Vázquez, G.; Colom, A. J. y Sarramona, J. (1994) Teoría de la Educación. Madrid, Taurus Universitaria.

COLÁs, M. ํㅗ. y BuendíA, L. (1998) Investigación educativa. Alfar, Sevilla. 
Colom, A. J. (1986) Pensamiento tecnológico y teoría de la educación, en Castillejo, J. L.; Colom, A. J.; Escámez, J.; García Carrasco, J.; Sanvicens, A.; Sarramona, J. y Vázquez. Tecnología y educación. Barcelona, Ceac, 13-30.

- (1997) La teoría de la educación: contexto actual de los estudios pedagógicos, en Colom, A. J. (coord.). Teorias e instituciones contemporáneas de la educación. Barcelona, Ariel, 146-169.

- (2002) La de(construcción) del conocimiento pedagógico: nuevas perspectivas en teoría de la educación. Barcelona, Paidós.

Colom, A. J. y Núñez Cubero, L. (2001) Teoría de la Educación. Madrid, Síntesis.

Colom, A. J. y Rodríguez, M. ${ }^{3}$ P. (1996) Teoría de la Educación y Ciencias de la Educación: carácter y situación, Teoría de la Educación. Revista Interuniversitaria, 8, 43-54.

Doval, L. y SANTos Rego, M. A. (1998) Educación e Neurociencia. Santiago de Compostela, Xunta de Galicia, Conselleria de Educación e Ordenación Universitaria, Dirección Xeral de Política Lingüística.

EsCámez J. (1986) Los valores en la pedagogía de la intervención, en CASTLLLejo, J. L.; Colom, A. J.; Escámez, J.; García Carrasco, J.; Sanvicens, A.; Sarramona, J. y Vázquez. Tecnología y educación. Barcelona, Ceac, 164-173.

Fermoso, P. (1982) Teoría de la educación. Una interpretación antropológica. Barcelona, Ceac.

Ferrero, J. J. (1994) Teoría de la Educación. Bilbao, Universidad de Deusto.

Fullat, O. (1991) Educación, en Altarejos, F.; Bouché, J.; Escámez, J.; Fullat; O.; Fermoso, P.;

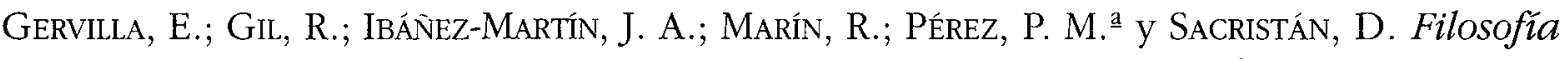
de la Educación Hoy. Conceptos. Autores. Temas. Madrid, Dykinson, 67-90.

García Aretro, L. (2000) La educación como fenómeno social, en Medina, R.; Rodríguez, T. y García, L. Teoría de la Educación II. Madrid, UNED, 97-112.

García Carrasco, J. (1983) Estudio preliminar, en Herbart, J. F. Pedagogia general derivada del fin de la educación. Barcelona, Humanitas (traducción de L. Luzuriaga).

- (1987) Apuntes de Teoría de la Educación. Tomo I. Salamanca, Ediciones de la Universidad de Salamanca.

- (1991) La Teoría de la Educación en la actividad académica española. Ponencia presentada en el XI Seminario Interuniversitario de Teoria de la Educación. Llanes, noviembrediciembre.

García Carrasco, J. y García del Dujo, A. (1995) Epistemología pedagógica (I), Teoría de la Educación. Revista Interuniversitaria, 7, 5-38.

- (1996) Teoría de la Educación. I. Educación y Acción Pedagógica. Salamanca, Universidad de Salamanca.

- (2001) Teoría de la Educación. II. Procesos primarios de formación del pensamiento y la acción. Salamanca, Universidad de Salamanca.

García Carrasco, J.; García del Dujo, A.; Barrón, A. y González, M. (1992) La Teoría de la Educación en la actividad académica española. Análisis de un indicador, Teoría de la Educación. Revista Interuniversitaria, 4, 41-52.

Gil Cantero, F. (1991) Sobre la posibilidad de un conocimiento autónomo de la educación y su aplicación a finalidades educativas. Teoria de la Educación. Revista Interuniversitaria, 3, 113-126.

Gimeno, J. (1983) El profesor como investigador en el aula: un paradigma de formación de profesores, Educación y Sociedad, 2, 51-73. 
Gimeno, J. y Pérez Gómez, A. (1992) Comprender y transformar la enseñanza. Madrid, Morata.

Ibáñez-Martín, J. A. (1992) La Filosofía de la Educación y el futuro de Europa, en Bárcena, F.; Gil, F.; IbáNiez-Martín, J. A.; Jover, G.; Ruiz Corbella, M. y Sacristán, D. La Filosofía de la Educación en Europa. Madrid, Dykinson, 11-21.

JoRdán, J. A. (1989) Teoría de la educación versus Filosofía de la Educación (Perspectivas actuales). Comunicación presentada al Symposion Internacional de Filosofia de l'educació. Barcelona.

LARROSA, J. (1990) El trabajo epistemológico en Pedagogía. Una propuesta constructivista. Barcelona, PPU.

López-Barajas, E. (2000) Fundamentos de metodología científica. Madrid, UNED (5 reimpresión, la 1 a edición es de 1988).

Marín, R. (1997) Teoría de la Educación, en Altarejos, F.; Bouché, J.; Escámez, J.; Fullat; O.; Fermoso, P.; Gervilla, E.; Gil, R.; Ibáñez-Martín, J. A.; Marín, R.; Pérez, P. M.ay Sacristán, D. Filosofia de la Educación boy. Diccionario filosófico-pedagógico. Dykinson, Madrid, 355-360.

Martínez, M. y BuXarrais, M. a R. (1992) La investigación en Teoría de la Educación, Teoría de la Educación. Revista Interuniversitaria, 4, 21-39.

MEDina, R. (1987) El enfoque tecnológico de la planificación curricular, en SARRAmOna, J. (ed.). Curriculum y Educación. Barcelona, Ceac, 95-111.

- (2000a) Explicación, norma y praxis en el conocimiento de la educación, en MEDINA, R.; Rodríguez, T. y GarCía, L. Teoría de la Educación I. Madrid, UNED, 157-175.

- (2000b) Estatuto epistemológico de la Teoría de la Educación, en MEDina, R.; Rodríguez, T. y García, L. Teoría de la Educación I. Madrid, UNED, 213-232.

- $(2000 \mathrm{c})$ Los procedimientos y técnicas lógico-formales en el acceso al conocimiento de la educación, en Medina, R.; Rodríguez, T. y García, L. Teoría de la Educación I. Madrid, UNED, 295-315.

Medina, R.; Rodríguez, T. y García, L. (2000) Teoria de la Educación I y II. Madrid, UNED. MOORE, T. W. (1980) Introducción a la teoría de la educación. Madrid, Alianza Universidad. MoRin, E. (1995) Introducción al pensamiento complejo. Barcelona, Gedisa.

NAssif, R. (1975) Pedagogia General. Buenos Aires, Kapelusz.

- (1980) Teoría de la Educación. Madrid, Cincel-Kapelusz.

Puig, J. M.a (1986) Teoría de la Educación. Una aproximación sistémico-cibernética. Barcelona, PPU.

- (1989): Sobre la Teoría de la Educación. Comunicación presentada al Symposion Internacional de Filosofia de l'Educació. Barcelona.

Quintana, J. M. a (1983) Pedagogía, Ciencia de la Educación y Ciencias de la Educación, en Basabe, J.; Colom, A. J.; Escolano, A.; García Carrasco, J.; Gutiérrez, G.; Palop, P.; QuintANA, J. M. y Rodríguez Diéguez, J. L. Estudios sobre epistemología y pedagogía. Madrid, Anaya, 35-57.

- (1988) Teoría de la educación. Concepción antinómica de la educación. Madrid, Dykinson.

Rodríguez Diéguez, J. L. (1985) Didáctica General. Madrid, Cincel.

Sáenz, O. (dir.) (1989) Didáctica General. Madrid, Anaya.

Sarramona, J. (1990) Tecnología educativa (Una valoración crítica). Barcelona, Ceac. 
- (1992) Justificación y sentido de una teoría de la educación, en Departamento DE Teoría DE la Educación y Pedagogía Social. Cuestiones actuales sobre educación. Madrid, UNED, 161-177.

- (1995) Fundamentos de la educación. Barcelona, Ceac.

- (2000) Teoría de la Educación. Reflexión y normativa pedagógica. Barcelona, Ariel.

Scheuerl, H. (1984) Sobre la cuestión de la fundamentación de las decisiones pedagógicas, Educación, 30, 78-94.

Stenhouse, L. (1984): Artistry and teaching: the teacher as focus of research and development, en HOPKINS, D. y WidEen, M. (eds.). Alternative perspectives on school improvement. Falmer Press, Lewes, 125-140.

Touriñán, J. M. (1987) Teoría de la Educación. Madrid, Anaya.

Trilla, J. (1987) Teoría de la educación (Bosquejo de una taxonomía), Cuadernos de Estudio, 8, 15-20.

Tusquets, J. (1972) Teoría de la Educación. Madrid, Magisterio Español.

VÁzQuez, G. (1994) Dimensión teórico-práctica de la educación, en CAstillejo, J. L.; VázQuez, G.; Colom, A. J. y Sarramona, J. Teoría de la Educación. Madrid, Taurus Universitaria, 4557.

Zabalza, M. A. (1997): Diseño y desarrollo curricular. Morata, Madrid. 